\title{
Formin-Dependent Synaptic Growth: Evidence That Dlar Signals via Diaphanous to Modulate Synaptic Actin and Dynamic Pioneer Microtubules
}

\author{
Catherine Pawson, Benjamin A. Eaton, and Graeme W. Davis \\ Department of Biochemistry and Biophysics, Programs in Neuroscience and Cell Biology, University of California, San Francisco, San Francisco, California \\ 94158-2822
}

The diaphanous gene is the founding member of a family of Diaphanous-related formin proteins (DRFs). We identified diaphanous in a screen for genes that are necessary for the normal growth and stabilization of the Drosophila neuromuscular junction (NMJ). Here, we demonstrate that diaphanous mutations perturb synaptic growth at the NMJ. Diaphanous protein is present both presynaptically and postsynaptically. However, genetic rescue experiments in combination with additional genetic interaction experiments support the conclusion that dia is necessary presynaptically for normal NMJ growth. We then document defects in both the actin and microtubule cytoskeletons in dia mutant nerve terminals. In so doing, we define and characterize a population of dynamic pioneer microtubules within the NMJ that are distinct from the bundled core of microtubules identified by the MAP1b-like protein Futsch. Defects in both synaptic actin and dynamic pioneer microtubules are correlated with impaired synaptic growth in dia mutants. Finally, we present genetic evidence that Dia functions downstream of the presynaptic receptor tyrosine phosphatase Dlar and the Rho-type GEF (guanine nucleotide exchange factor) trio to control NMJ growth. Based on the established function of DRFs as Rho-GTPase-dependent regulators of the cell cytoskeleton, we propose a model in which Diaphanous links receptor tyrosine phosphatase signaling at the plasma membrane to growth-dependent modulation of the synaptic actin and microtubule cytoskeletons.

Key words: Drosophila; neuromuscular junction; formin; GTPase; Liprin; phosphatase

\section{Introduction}

Individual neurons acquire stereotyped morphologies during development. It is well established that signaling molecules including the neurotrophins, growth factors, and morphogens can profoundly influence the global growth and survival of individual nerve cells (Aberle et al., 2002; Marqués, 2005; Zweifel et al., 2005; Bamji et al., 2006; Reichardt, 2006). There is also evidence that local, intercellular signaling systems participate in defining synaptic and dendritic arborizations (Luo and O'Leary, 2005). Although considerable progress has been made, the identity of the intercellular signaling systems, and how these signals are coupled to the modulation of the underlying neuronal cytoskeleton, remains to be elaborated.

The importance of the presynaptic actin and microtubule cytoskeletons during synapse morphogenesis and growth of the Drosophila neuromuscular junction has been established through the analysis of mutations in essential cytoskeletal regulatory pro-

Received Aug. 29, 2008; accepted Sept. 6, 2008.

This work was supported by a National Institutes of Health National Research Service Award (B.A.E.) and Nationa Institutes of Health Grant NS047342 (G.W.D.).

Correspondence should be addressed to Graeme W. Davis at the above address. E-mail: gdavis@biochem.ucsf.edu.

B. A. Eaton's present address: Department of Physiology, Barshop Institute of Longevity and Aging Studies, University of Texas Health Sciences Center, San Antonio, TX 78229.

DOI:10.1523/JNEUROSCI.0833-08.2008

Copyright $\odot 2008$ Society for Neuroscience $\quad$ 0270-6474/08/2811111-13\$15.00/0 teins. For example, perturbation of the presynaptic spectrin-actin skeleton profoundly disrupts neuromuscular junction (NMJ) growth and stability (Pielage et al., 2005, 2006). Additional actin regulatory proteins implicated in synaptic growth in Drosophila include the NWASP (neuronal Wiskott-Aldrich syndrome protein) interacting protein nervous wreck $(N w k)$, the dynaminassociated protein Dap160/Intersectin, and N-ethylmaleimidesensitive factor (NSF) (Stewart et al., 2002; Coyle et al., 2004; Marie et al., 2004; Stewart et al., 2005). The integrity of a stable population of bundled presynaptic microtubules at the Drosophila NMJ is also required for normal synaptic growth (Roos et al., 2000). Presynaptic bundled microtubules (MTs) are labeled by the MAP1b-like protein Futsch and futsch mutations disrupt both NMJ growth and synaptic microtubule organization (Roos et al., 2000). Several additional signaling systems have been shown to be required for the normal integrity of presynaptic microtubules including Vap33A (Pennetta et al., 2002), aPKC (atypical protein kinase C) (Ruiz-Canada et al., 2004), spastin (Sherwood et al., 2004), sec8 (Liebl et al., 2005), and protein phosphatase 2A (Viquez et al., 2006).

Despite this progress, there is not a clear picture for how the synaptic MT and actin cytoskeletons are regulated by intercellular, synaptic growth-related signaling at the Drosophila NMJ. The formins are a highly conserved family of proteins with well established functions during the assembly and organization of the actin and microtubule cytoskeletons (Palazzo et al., 2001; Wallar 
and Alberts, 2003; Eng et al., 2006). Although the formins have been implicated in diverse cellular processes, little is known about formin protein function within the nervous system. Here, we provide evidence that a Drosophila formin encoded by diaphanous is an essential molecular effector that conveys signaling from a presynaptic receptor protein tyrosine phosphatase (Dlar) to the underlying actin and microtubule cytoskeletons. We also show that this signaling is necessary for normal synaptic growth. Finally, in so doing, we also characterize a population of dynamic, pioneer synaptic microtubules and provide evidence for Diaphanous-dependent regulation of these pioneer microtubules.

\section{Materials and Methods}

Generation of UAS-EB1-GFP transgenic Drosophila. C-terminal fusion of green fluorescent protein with Drosophila EB1 (EB1-GFP) was obtained from Drs. Steve Rogers and Ron Vale (University of California, San Francisco, San Francisco, CA) (unpublished data) and cloned into the pUAST vector using standard methodology. Transgenic Drosophila lines were obtained with homozygous viable UAS-EB1-GFP transgenes inserted on the second and third chromosomes.

Cell culture. All Drosophila S2 cell protocols were performed as described in the Drosophila Expression System Manual (Stratagene). Briefly, Drosophila S2 cells, stably expressing the GAL4 transcription factor, were cultured in Schneider's medium supplemented with $10 \%$ fetal calf serum at $25^{\circ} \mathrm{C}$. For RNA-mediated knockdown of Diaphanous, cells were incubated in serum-free media with $19 \mu \mathrm{g}$ of double-stranded RNA (dsRNA) against Dia. Cells were incubated in RNA for $5 \mathrm{~d}$ before transfection with the pUAST-EB1-GFP plasmid using a standard calcium phosphate transfection protocol. Cells were incubated for $48 \mathrm{~h}$ before plating on ConA ( $0.5 \mathrm{mg} / \mathrm{ml}$ stock; Sigma-Aldrich)-coated coverslips for live imaging and subsequent analysis (Rogers et al., 2002).

Genetics. Drosophila were maintained at $25^{\circ} \mathrm{C}$ on normal food. The following strains were used in this study: $\mathrm{dia}^{2} / C y O-G F P, D f(2 L) T W 2 /$ CyOGFP, dlar ${ }^{5.5} / C y o-G F O, U A S-H A-D i a[\Delta N \Delta C] / T M 6$ (gift from Purnilla Rorth, Temasek Life Sciences Laboratory, National University of Singapore, Singapore), dlar ${ }^{13.2} / \mathrm{CyO}$-GFP, trio $^{1} / \mathrm{TM} 6$, trio $^{8} / \mathrm{Tm} 6 \mathrm{~b}, \mathrm{abl} \mathrm{l}^{1} /$ TM6, chic $^{21} / C y O-G F P$, UAS-EB1-GFP, UAS-GFP-actin. For CNS expression of UAS-EB1-GFP, or UAS-GFP-actin, flies were crossed to one of three GAL4 lines: (1) elaV ${ }^{c 155}$-GAL4 (pan neuronal), (2) OK6-GAL4 (motor neuron specific) (Aberle et al., 2002), and (3) D42-GAL4 (CNS specific). Pickpocket-Gal4 was used to drive expression specifically in the PNS (unpublished reagent) (generous gift from C. Yang, W. B. Grueber, L. Y. Jan, and Y. N. Jan, University of California, San Francisco, San Francisco, CA).

Live imaging and kymograph analysis. Wandering third-instar larvae were dissected in $\mathrm{HL} 3$ saline $\left(0.5 \mathrm{~mm} \mathrm{Ca}^{2+}\right)$ and stably positioned on a glass coverslip using pressure pins held in place using a magnetic surface surrounding the glass slide. EB1-GFP-labeled MTs were imaged using a GFP filter set (Chroma) with a $100 \times$ Plan-Apochromat (0.97 numerical aperture) oil-immersion objective (Zeiss). Images were acquired with a Photometrics CoolSnap HQ cooled CCD camera (Roper Scientific) and Kodak EEV57 back thinned chip (Roper Scientific) mounted on an inverted microscope (Zeiss Axiovert 200). The sample was illuminated using a $100 \mathrm{~W}$ xenon light source (Sutter Instrument) and liquid light guide (Sutter Instrument). Images were acquired with a $500 \mathrm{~ms}$ exposure and $750 \mathrm{~ms}$ frame rate. Images were collected at room temperature $\left(18^{\circ} \mathrm{C}\right)$ for a maximum of $1 \mathrm{~h}$ per preparation. Imaging was controlled by Slidebook software (Intelligent Imaging Innovations).

To generate kymographs for rate analysis, axons in the segmental nerve overlaying muscle 4 were imaged, and the images were deconvolved to remove background fluorescence. A 1-pixel-wide mask was drawn over a length of axon in the first time point this mask was copied to all subsequent frames. The smooth curve analysis function of SlideBook 4 was applied to these masks to generate the kymographs. This function aligns the masked regions from each time point. Any movement that occurs in the masked region over the time imaged appears as a diagonal line through the kymograph. The slope of this line was mea- sured using Slidebook software to determine the rate of movement. The synapse on muscle 4 was selected for imaging and analysis because it was possible to image several boutons in the same plane of focus. Puncta in the synapse often took a curved path in a bouton. To generate kymographs for the synapse, a mask was created over the specific path of an individual puncta, resulting in a curved line for many of the masks. These masks were then converted to kymographs using the smooth curve analysis function of Slidebook software. For a given segment of axon or synapse, all puncta that remained in the plane of focus for at least five frames were analyzed.

Pharmacology. Latrunculin A was dissolved in DMSO to a concentration of $1 \mathrm{~mm}$ to create a stock. The latrunculin A stock solution was diluted to a range of concentrations in HL3 saline $\left(0.5 \mathrm{mM} \mathrm{Ca}^{2+}\right)$. Wandering third-instar larvae were dissected in HL3 $\left(0.5 \mathrm{mM} \mathrm{Ca}^{2+}\right)$ and incubated for $15 \mathrm{~min}$ at room temperature in either DMSO (control) or latrunculin A (experimental). Larvae were then imaged for GFP-actin or EB1-GFP.

Antibody staining. Primary antibodies used in this study include monoclonal antibody (mAb) 22C10 (1:50; obtained from the Developmental Studies Hybridoma Bank), Cy3- and FITC-conjugated HRP (1: 200; obtained from MP Biomedicals/Cappel), and anti-brp and mAb 3C11 (1:10; obtained from Developmental Studies Hybridoma Bank). Secondary antibodies used were FITC-labeled anti-mouse (1:200) and Cy3-conjugated anti-mouse (1:200). All secondary antibodies were obtained from MP Biomedicals/Cappel.

\section{Results}

Formins have been shown to participate in the nucleation of unbranched actin filaments by association with the barbed end of actin filaments (Pruyne et al., 2002; Zigmond et al., 2003; Romero et al., 2004). In this capacity, the formins have been implicated in the formation and modulation of actin cables, stress fibers, and actin-rich cell adhesions (Watanabe et al., 1999; Ishizaki et al., 2001; Kovar et al., 2003; Kobielak et al., 2004; Zigmond, 2004). A subfamily of formins, the Diaphanous-related formins (DRFs), have also been shown to bind microtubule plus ends and function with adenomatous polyposis coli (APC) to control microtubule stabilization (Palazzo et al., 2004; Wen et al., 2004). In Drosophila, diaphanous encodes a prototypical DRF protein.

We identified mutations in the diaphanous gene in a largescale screen for genes that are required for the normal growth and/or stability of the Drosophila NMJ. The basis of this forward genetic screen is direct visualization of the Drosophila NMJ in dissected third-instar larvae. For each independent mutant or gene-specific RNA interference (RNAi), we directly visualize juxtaposed presynaptic and postsynaptic compartments at the thirdinstar NMJ. The presynaptic nerve terminal is stained with either anti-synapsin (vesicle-associated protein) or anti-Brp (labeling the presynaptic active zone) in combination with anti-Dlg to label the postsynaptic muscle membrane folds. We examine 30-50 NMJs from at least three independent animals per mutant or gene-specific RNAi and score each for a change in bouton number (an assay for synapse growth) or the increased presence of synaptic retraction events [an assay for synapse stability as defined in previous publications (Eaton et al., 2002; Eaton and Davis, 2005; Pielage et al., 2005)]. The diaphanous gene was identified as being required for normal NMJ growth without an effect on synapse stability.

\section{Diaphanous is present both presynaptically and postsynaptically at the NMJ}

Diaphanous is a classical formin with two formin homology domains (FH1 and FH2) (Fig. $1 B$ ). The FH1 domain is a prolinerich domain that binds $\mathrm{SH} 3$ - and WW-containing proteins such as profilin (Sagot et al., 2002; Higgs, 2005). The C-terminal FH2 

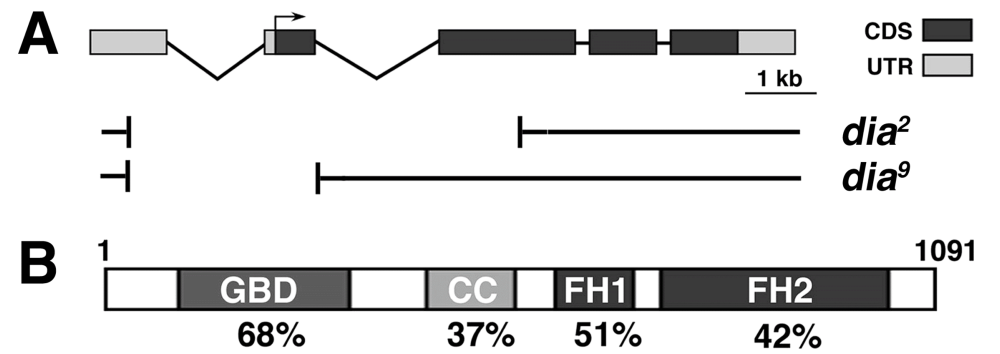

C
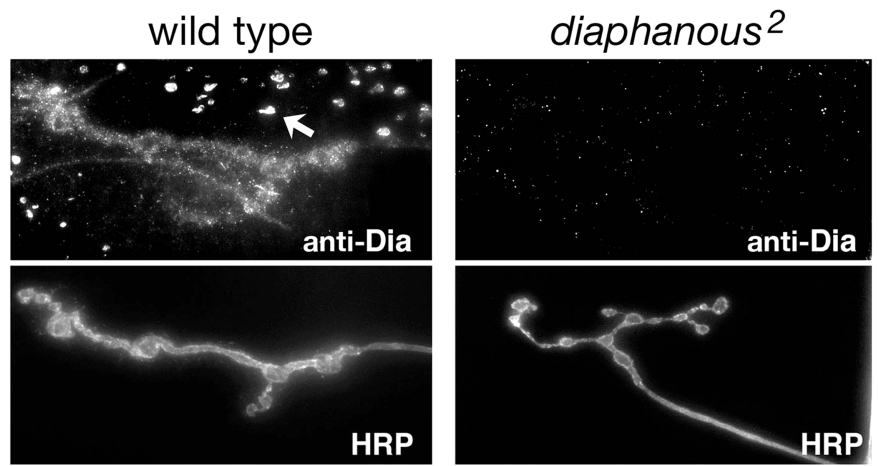
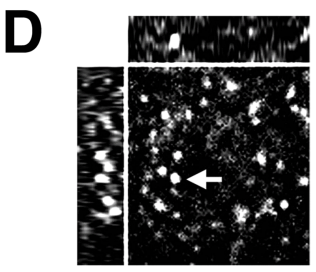

anti-Dia

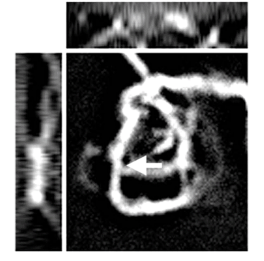

anti-Futsch

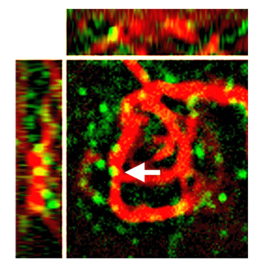

Figure 1. Diaphanous is present presynaptically and postsynaptically. $\boldsymbol{A}$, Diagram of the Diaphanous locus with protein coding sequences (CDS) and untranslated sequences (UTR) indicated. Small deletions associated with the dia $^{2}$ and dia $^{9}$ mutants are shown (Castrillon and Wasserman, 1994). B, Diagram of Dia protein including the GBD, the coiled-coil region (CC), and the formin homology domains 1 and 2 (FH1 and FH2, respectively). The percentage amino acid sequence identity between Drosophila Dia protein and the mouse Dia2 protein (mDia2) is indicated. C, Immunofluorescent image of a third-instar NMJ from wild-type (left two panels) and a dia ${ }^{2}$ larva (right two panels) stained with anti-Dia (top) and anti-HRP (bottom). The arrow indicates Dia staining of putative endosomes postsynaptically. Note that staining is nearly absent at the dia ${ }^{2}$ mutant NMJ. $\boldsymbol{D}$, Three-dimensional imaging demonstrates colocalization of Dia protein (left panel) and the Futsch protein (center panel) within a single presynaptic bouton. The right panel represents a merged image of the staining of the Dia and Futsch proteins. The arrow indicates Dia protein closely associated with presynaptic Futsch bound microtubules. The insets above represent the $z$-axis cut as a horizontal line through the indicated Dia puncta (arrow). The insets to the left represent the $z$-axis cut as a vertical line through the same puncta.

domain is known to make contacts with actin and is required for protein dimerization (Higgs, 2005). Together, the FH1 and FH2 domains mediate the actin regulatory abilities common to formin proteins (Sagot et al., 2002; Kovar and Pollard, 2004; Romero et al., 2004; Higgs, 2005; Pellegrin and Mellor, 2005). In addition to the classical formin domains, Diaphanous also contains an N-terminal Rho-type GTPase binding domain (GBD) and a C-terminal autoinhibitory domain (Alberts, 2001; Higgs, 2005; Wallar et al., 2006). These domains are typical of the DRF subfamily of formin proteins. The C-terminal autoinhibitory domain binds to a sequence within the N-terminal GBD and is thought to prevent the interaction of profilin and g-actin with the FH1 and FH2 domains. Binding of active Rho-GTPases to the GBD is thought to relieve the association of the autoinhibitory domain with the $\mathrm{N}$ terminus, allowing the DRF to become active (Alberts, 2001; Wallar et al., 2006).

Previous genetic studies of diaphanous in Drosophila have identified mutations in the dia gene including the $\mathrm{dia}^{2}$ and $\mathrm{dia}^{9}$ alleles (Fig. $1 \mathrm{~A}$ ). The $\mathrm{dia}^{2}$ mutation lacks the entire first exon and deletes one-half of the second coding exon. Previously published merge

genetic data are consistent with the conclusion that $\mathrm{dia}^{2}$ is a null mutation (Castrillon and Wasserman, 1994). The $\mathrm{dia}^{2}$ homozygous animals are early larval lethal, although some larvae survive to the third-instar stage, allowing analysis of synaptic growth and function. The $\mathrm{dia}^{9}$ allele lacks a portion of the first exon and is considered to be a hypomorphic mutation (Castrillon and Wasserman, 1994). These animals survive to late third-instar stages.

We first addressed whether Dia protein is present at the third-instar larval NMJ. In wild-type third-instar larvae, anti-Dia staining was observed to concentrate at the NMJ (Fig. 1C). Some staining is clearly postsynaptic, localizing to punctate structures in the muscle that may represent endosomes based on their size and distribution (Fig. $1 C$, arrow). Additional staining overlaps the presynaptic nerve terminal. The synaptic and putative endosomal staining are both eliminated in the $d i a^{2}$ null mutant background, demonstrating that this staining pattern represents the distribution of Dia protein at the NMJ (Fig. 1C). We pursued additional experiments to investigate whether Dia protein might reside within the presynaptic nerve terminal. To do so, we costained the NMJ for Dia and the presynaptic-specific microtubule-associated protein Futsch (Roos et al., 2000). Three-dimensional reconstruction of the nerve terminal demonstrates that a portion of the Diaimmunoreactive puncta reside in close association with bundled presynaptic microtubules (Fig. 1D). A similar conclusion was made after analysis of the NMJ that was costained with Dia and the presynaptic membrane marker antiHRP (data not shown). Thus, we conclude that Dia is present both presynaptically and postsynaptically at the Drosophila NMJ.

Presynaptic Dia is necessary for normal synaptic growth We identified dia mutations in a screen based on a decrease in synaptic bouton number at muscles 6/7 (Fig. 2A,B). This decrease in bouton number is also observed at muscle 4 (Fig. 2C,D), demonstrating a general requirement of dia during NMJ growth. We have focused our analysis on muscle 4 because this NMJ is typically positioned in the central, flattest region of the muscle and is, therefore, ideally suited for live imaging studies of the synaptic actin and microtubule cytoskeletons (see below). Quantification of bouton numbers at muscle 4 confirms a significant decrease in bouton number at the dia null and hypomorphic NMJs compared with wild type ( $\mathrm{dia}^{2} / \mathrm{Df}$, $65.64888 \pm 2.256 \%$; $\mathrm{dia}^{2} / \mathrm{dia}^{2}, 64.744 \pm 2.4956 \% ; \mathrm{dia}^{2} / \mathrm{dia}^{9}, 69.8213 \pm 2.86946 \%$; data are percentage wild-type bouton number) (Fig. $2 F$ ). It has been shown that muscle growth is coupled to growth of the presynaptic nerve terminal at the Drosophila NMJ (Aberle et al., 2002). To determine whether the decrease in bouton number 
observed in the dia mutants was attributable to a decrease in muscle size, we measured muscle size in each mutant background. There is no significant decrease in muscle size in any of the tested mutations compared with wild type (data not shown). However, we observe a significant $15 \%$ increase in muscle size in the dia mutant, which would cause us to underestimate the bouton-to-muscle surface ratio. Thus, we conclude that the observed changes in bouton number reflect impaired presynaptic nerve terminal growth. Finally, we measured bouton areas in the dia mutant background because impaired bouton numbers are often associated with a change in bouton morphology. We find a highly significant increase in average bouton area in the dia mutant background (wild type, $4.96 \pm 0.15 \mu \mathrm{m}^{2}, n=725 ; \mathrm{dia}^{2}$, $\left.12.6 \pm 0.49 \mu \mathrm{m}^{2} ; p<0.001 ; n=334\right)$, consistent with observed changes in NMJ morphology in other mutations such as the receptor tyrosine phosphatase dlar.

Because Diaphanous protein is present both presynaptically and postsynaptically, we next addressed whether Diaphanous is required in the presynaptic neuron or postsynaptic muscle cell for normal synaptic growth. To do so, we expressed a diaphanous rescue transgene (UAS-dia ${ }^{a c t}$ ) in the background of the $d i a^{2}$ null mutant background. The UAS-dia ${ }^{a c t}$ transgene lacks both the N-terminal GBD and the C-terminal DAD domains. Based on work in other systems, this transgene is predicted to behave as a constitutively active Diaphanous molecule, allowing us to increase Dia function independent of the signaling systems necessary for activation of the Dia protein (Watanabe et al., 1999; Tominaga et al., 2000; Somogyi and Rørth, 2004; Williams et al., 2007). We find that overexpression of UAS-dia ${ }^{a c t}$ in either the nerve or the muscle in a wild-type background has no effect on synaptic growth (data not shown). However, neuronal expression of UAS-dia ${ }^{a c t}$ is sufficient to rescue synaptic growth in the $\mathrm{dia}^{2}$ null mutant background (Fig. $2 E, F$ ). It is possible that nonspecific actions of the activated dia transgene contribute to genetic rescue. However, we believe that the specificity of the observed rescue, restoring the NMJ to wild-type size without causing a neomorphic alteration in NMJ morphology, makes this possibility unlikely. Therefore, we conclude that Diaphanous is required presynaptically for normal NMJ growth.

A second, prototypical formin is encoded by the cappuccino gene in Drosophila (Emmons et al., 1995). We also examined mutations in capp to determine whether synaptic growth might be generally perturbed by altered formin signaling. However, quantification of synaptic bouton numbers demonstrates that capp is not required for NMJ growth (Fig. $2 F$ ). Additionally, synapse morphology is normal in capp (data not shown). These data highlight the fact that dia mutations were identified ran- domly in a forward screen for mutations that disrupt synaptic growth.

\section{Dia is necessary for the maintenance of the presynaptic} actin cytoskeleton

Dia and other formin proteins have been shown to influence actin polymerization. Therefore, we asked whether impaired synaptic growth observed in the dia mutant background is correlated with a change in the presynaptic actin cytoskeleton. To visualize the presynaptic actin cytoskeleton, we expressed a UAS-actinGFP transgene presynaptically in the $\mathrm{dia}^{2}$ mutant background. Consistent with a recent study (Nunes et al., 2006), we find that presynaptic actin at the Drosophila NMJ is organized into discreet foci that are distributed throughout the NMJ (Fig. $3 A, C, E$ ). Postfixation staining of the presynaptic nerve terminal membrane allowed us to quantify the distribution of these presynaptic actin puncta within individual synaptic boutons (Fig. $3 B, D, F$ ). Quantification of the number of actin foci per bouton reveals a $\sim 50 \%$ decrease in the number of organized actin foci at the dia NMJ compared with wild type (wt, $5.51 \pm 0.2$ foci/bouton, $n=325$; 

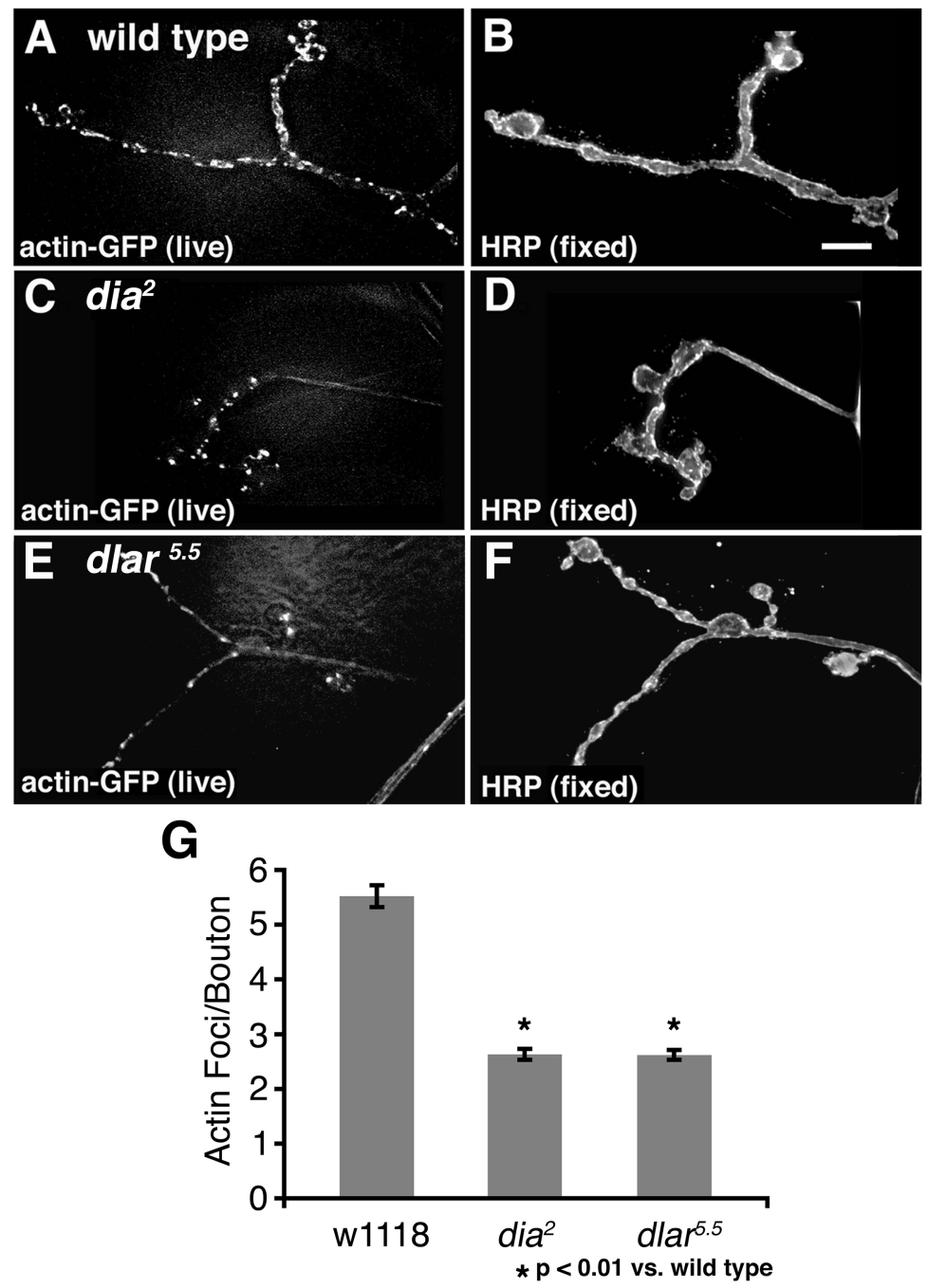

Figure 3. Diaphanous regulates the presynaptic actin cytoskeleton. Representative projections from larval NMJs expressing an actin-GFP transgene in a wild type $(\boldsymbol{A}, \boldsymbol{B})$, a dia ${ }^{2 / 2}$ mutant animal $(\boldsymbol{C}, \boldsymbol{D})$, and a dlar ${ }^{5.5}$ mutant animal $(\boldsymbol{E}, \boldsymbol{F})$. Images show either a live image of actin-GFP or the same NMJ after fixation and staining with anti-HRP to reveal the presynaptic membrane, as indicated. G, Graph represents the number of synaptic actin foci divided by the number of boutons. Error bars represent SEM. Scale bar, $10 \mu \mathrm{m}$. Asterisks indicate statistically significant differences compared with wild type.

$\mathrm{dia}^{2} / \mathrm{dia}^{2}, 2.64 \pm 0.1$ foci/bouton, $n=369 ; p<0.001$ ) (Fig. 3G). We also visualized presynaptic actin foci by expressing a GFPtagged actin binding protein (Edwards et al., 1997). This transgene controlled for possible artifacts arising from actin overexpression. When the NMJs of larvae expressing this transgene were imaged, the GFP pattern was indistinguishable from that observed using the UAS-actin-GFP (data not shown). These data suggest that loss of Dia within the presynaptic nerve terminal leads to impaired actin polymerization or stabilization and, as a consequence, a decrease in the steady-state levels of polymerized actin. This defect could reasonably account for impaired synaptic growth. To investigate this possibility further, we pursued genetic epistasis experiments with genes known to participate in the regulation of neuronal actin.

Genetic evidence that Dia functions downstream of Dlar and Trio during NMJ growth

The dia mutant phenotype is manifest as a decrease in synaptic bouton number with a corresponding increase in bouton area. A similar phenotype has been documented at the NMJ of the dlar mutant animals. The dlar gene encodes a receptor tyrosine phos- phatase that has been shown to signal via an associated Rho-GEF encoded by trio to control motor axon guidance (Krueger et al., 1996; Bateman et al., 2000; Kaufmann et al., 2002). In contrast, loss of other putative actin-regulatory proteins present at the Drosophila NMJ such as NSF (Stewart et al., 2002, 2005), Nervous-wreck (Coyle et al., 2004), Dap160/Intersectin (Marie et al., 2004), and Profilin (our unpublished data) cause a dramatic change in synapse morphology including an increase in the formation of small, highly branched synaptic boutons termed satellite boutons. Thus, the phenotypic similarity of the dlar and dia mutations suggested that these genes might function together to control synaptic growth. Furthermore, by analysis with DRF function in other systems, it is reasonable to hypothesize that trio could signal via a GTPase that subsequently activates Dia to mediate a change in the underlying synaptic cytoskeleton necessary for synaptic growth (Watanabe et al., 1999; Li and Higgs, 2003, 2005; Ridley, 2006; Wallar et al., 2006). Therefore, we tested this hypothesis genetically by analyzing double mutant combinations of dia with dlar and trio.

First, we demonstrate that heterozygous null mutations in dia as well as dlar and trio do not significantly impair synaptic growth at the NMJ. Indeed, the dlar heterozygous animals show a small, but statistically significant, increase in bouton number (Fig. $4 A, B, G$ ). We also confirmed previous results demonstrating that homozygous dlar mutant NMJ have significantly fewer synaptic boutons compared with wild type (data not shown) (Kaufmann et al., 2002). Next, we analyzed bouton number in animals that harbor double heterozygous mutations by combining $\mathrm{dia}^{2} /+$ with either dlar $^{5.5} /+$ (Fig. $4 E, G$ ) or trio $^{1} /+$ (Fig. $4 D, G$ ). In both cases, the double heterozygous mutant combinations result in a statistically significant decrease in synaptic bouton number (Fig. 4G) $(p<$ 0.01 ). Finally, we asked whether the genetic interaction between trio and dlar, first identified in the context of Drosophila axon guidance, also occurs when NMJ growth is assayed (Krueger et al., 1996; Bateman et al., 2000). We find that the double heterozygous mutant combination of $\mathrm{dlar}^{5.5} /+$; rio $^{1} /+$ resulted in a significant decrease in NMJ size, similar to that seen for the dia genetic combinations (Fig. $4 F, G$ ). Together, these genetic data indicate that dia, dlar, and trio all function in the process of NMJ growth and could function as a single signaling pathway. To further explore this possibility, we generated animals that are double homozygous mutations of dia and dlar. If these genes function in the same pathway, then we expect that the double mutant will be no more severe than the $\operatorname{dia}^{2}$ null mutation alone. Analysis of the $d i a^{2}-d l a r^{5.5}$ double mutants reveals a decrease in synaptic bouton number that is not significantly different from either single mutation alone (Fig. 4C,G). Furthermore, when we image actin-GFP at the NMJ of the $d l a^{5.5}$ mutants, we find a defect that is quanti- 
tatively identical to that observed in the dia mutant background (Fig. 3). These same experiments could not be repeated with trio because it is a homozygous lethal mutation. Together, these data lend support to the conclusion that Diaphanous functions with Dlar and Trio to control synaptic growth. Because Dia is a Rho-GTPase effector protein, we propose that Dia functions downstream of Trio to control NMJ growth. Finally, because Dlar and Trio are expressed only presynaptically at the NMJ (Newsome et al., 2000; Kaufmann et al., 2002), these genetic data also provide additional evidence that Dia functions presynaptically to specify NMJ growth.

If Dia functions downstream of Dlar and is primarily responsible for mediating the growth-related function of the Dlar receptor, then we might expect that expression of the activated Dia transgene (UAS$\mathrm{dia}^{a c t}$ ) would be able to rescue the NMJ growth defects observed in the Dlar mutant. Remarkably, neuronal expression of $U A S$-dia ${ }^{a c t}$ not only rescues boutons numbers in the Dlar mutant (supplemental Fig. 1, available at www.jneurosci.org as supplemental material), but also significantly improves several measures of adult health and viability. In this experiment, bouton numbers were, once again, statistically significantly decreased in dlar mutant compared with wild type at muscle 4 (56.9 \pm $2.8 \%$ of wild type; $n=22 ; p<0.001)$ and expression of UAS-dia ${ }^{a c t}$ in the dlar mutant significantly increased bouton numbers toward wild-type values (86.29 \pm $3.27 \%$ of wild type; $n=22$; the rescue of bouton number is significant compared with dlar alone, $p<0.001)$. This experiment was repeated with quantitatively identical results at muscles $6 / 7$ (dlar ${ }^{5.5} /$ dlar ${ }^{13.2}, 41.0 \pm 3.98 \%$ of wild type, $n=18$; and expression of UAS-dia ${ }^{a c t}$ in dlar $r^{5.5} /$ dlar $^{13.2}, 85.77 \pm 5.04 \%$ of wild type, $n=18$; the rescue of bouton number is significant compared with dlar alone, $p<0.001)$. There is no change in statistical significance if the bouton counts are corrected for muscle size. In addition, dlar mutants eclose into adults at a lower rate compared with genetic controls and those dlar mutants that do eclose, do so with severely impaired health and fail to inflate their wings (supplemental Table 1, available at www.jneurosci.org as supplemental material). In contrast, expression of UAS-dia act in the dlar mutant increases the percentage of animals that eclose to adults. Furthermore, a much higher percentage of these animals inflate their wings. Together, these data provide additional genetic evidence that Dia functions downstream of Dlar during neural development and during NMJ growth.

Evidence in other systems indicates that Diaphanous can function together with additional components of the cellular machinery necessary for actin polymerization including profilin. Therefore, we also tested for genetic interactions between $\mathrm{dia}^{2}$ and profilin and the tyrosine kinase $a b l$. However, we did not uncover evidence of a transheterozygous interaction between dia and either profilin/+ or $a b l /+$. The early lethality of both genes prevented us from assaying synaptic growth in double homozygous mutations. These data are inconclusive regarding how Dia might interact with the actin regulatory machinery. However, these data serve to highlight the significance of the observed transheterozygous interaction between dia and both dlar and trio.

\section{Characterization of a dynamic population of presynaptic pioneer microtubules at the NMJ}

Diaphanous-related formin proteins have also been shown to modulate the dynamic behavior of microtubules. For example, activated mDial and mDia2 lead to the formation of a stable population of microtubules labeled by glu-tubulin, whereas inhibition of mDia activity prevents the generation of this population of microtubules in cell-wounding and migration assays (Palazzo et al., 2001, 2004; Wen et al., 2004; Eng et al., 2006). In mammalian cell culture studies, these effects on microtubules are independent of the Dia-dependent modulation of actin polymerization. Therefore, we sought to investigate the function of Dia on the synaptic microtubule cytoskeleton.

As highlighted previously, several studies have shown that dis- 
ruption of a stable core of bundled microtubules at the NMJ is correlated with a defect in synaptic growth (Roos et al., 2000). However, Dia has been shown to influence dynamic microtubules through signaling at the microtubule plus ends. Although a dynamic population of microtubules has been imaged within dendrites and axons of Drosophila neurons (Rolls et al., 2007), this population of microtubules has yet to be characterized in detail within the presynaptic nerve terminal. A recent study has examined the fluorescence recovery after photobleaching for GFP-tagged tubulin and provided evidence for the existence of dynamic presynaptic microtubules (Yan and Broadie, 2007). Here, we first set out to image and quantify this dynamic population of presynaptic microtubules at the Drosophila NMJ.

The expression of GFP-tagged EB1 protein has been used to visualize the plus ends of individual growing MTs in vitro (Morrison et al., 2002; Ma et al., 2004; Piehl et al., 2004; Wen et al., 2004). EB1 binds preferentially to a site within the GTP cap of growing MT plus ends providing a punctate, live marker of the MT plus end that is suitable for quantitative live imaging of microtubule movement and polymerization (Mimori-Kiyosue et al., 2000; Schuyler and Pellman, 2001; Rogers et al., 2002; Tirnauer et al., 2002). Because EB1-GFP binds preferentially to the growing MT plus end, it selectively marks a dynamic population of MTs undergoing plus end polymerization (Mimori-Kiyosue et al., 2000; Rogers et al., 2002; Tirnauer et al., 2002). Low levels of EB1-GFP also decorate the length of MTs through a mechanism that is thought to be distinct from the preferential plus end binding (Tirnauer et al., 2002). We have adapted the use of EB1-GFP to quantify the movement of a dynamic population of axonal and synaptic MT in Drosophila axons and the neuromuscular synapse, in vivo.

We first demonstrate that expression of Drosophila EB1-GFP marks the plus ends of growing MTs, in vitro, in a manner that is quantitatively and qualitatively similar to previous work in mammalian cell systems (supplemental Fig. 2 and supplemental Movie 1, available at www.jneurosci.org as supplemental material) (Morrison et al., 1998; Mimori-Kiyosue et al., 2000; Morrison et al., 2002; Tirnauer et al., 2002). In cultured Drosophila S2 cells, EB1-GFP puncta generally originate at the center of the cell, presumably at the microtubule organizing center, and these puncta move toward the cell periphery as has been observed in other systems (supplemental Movie 1, available at www. jneurosci.org as supplemental material) (Morrison et al., 1998; Mimori-Kiyosue et al., 2000; Tirnauer et al., 2002). Each puncta is a defined spot present at the MT plus end with a characteristic comet tail consistent with previous work examining EB1 protein in S2 cells (Rogers et al., 2002). This characteristic comet tail reflects the gradual release of EB1-GFP along the length of the growing MT as the GTP cap is gradually hydrolyzed (Rogers et al., 2002; Tirnauer et al., 2002; Stepanova et al., 2003; Wen et al., 2004). We measured the rate of EB1-GFP puncta movement in the S2 cells and find that the rate of movement $(6.62 \pm 0.17$ $\mu \mathrm{m} / \mathrm{min}$ ) (supplemental Fig. 2, available at www.jneurosci.org as supplemental material) is similar to that observed in other in vitro cell types (Tanaka et al., 1995; Tirnauer et al., 2002). With this as a background, we generated transgenic Drosophila harboring the EB1-GFP transgene under UAS control to quantify the behavior of MT plus end movement within Drosophila axons and synapses in vivo.

We first examined the movement of EB1-GFP puncta in axons within the segmental nerve of third-instar Drosophila larvae (supplemental Movie 2 and supplemental Fig. 3, available at www. jneurosci.org as supplemental material). When UAS-EB1-GFP is expressed pan-neuronally using the elaV-GAL4 $4^{c 155}$ driver, individual EB1-GFP puncta can be observed moving both toward the periphery and toward the CNS (supplemental Movie 2, available at www.jneurosci.org as supplemental material). Because the segmental nerve contains both motoneurons (cell body in the CNS) and sensory neurons (cell body in the periphery), it is not possible, using this GAL4 driver, to determine the orientation of MT plus ends within individual axons. Therefore, we turned to cell type-specific GAL4 lines that allow us to drive UAS-EB1-GFP selectively in motoneurons (OK6-GAL4) or sensory neurons ( $p$ pk-GAL4). When OK6-GAL4 was used, all of the EB1-GFP puncta moved in the same direction, from the CNS toward the periphery (data not shown). When $p p k-G A L 4$ was used, we observe that all EB1-GFP puncta move from the periphery toward the CNS (data not shown). Thus, dynamic MTs within the axons of sensory and motoneurons are uniformly oriented with their plus ends directed away from the cell body toward the synapse.

We next quantified the rate of EB1-GFP movement within motoneuron axons in vivo. For these experiments, $>100$ individual EB1-GFP puncta per preparation were analyzed, and data were acquired from at least 10 different animals were measured for each genetic combination. For each EB1-GFP puncta, the rate of puncta movement was determined by kymograph analysis (supplemental Fig. 3, available at www.jneurosci.org as supplemental material) (see Materials and Methods). Using this method of quantification, we calculate that EB1-GFP puncta move at a rate of $4.1 \mu \mathrm{m} / \mathrm{min}$ within axons of Drosophila motoneurons. In these experiments, we also controlled for possible effects of EB1-GFP expression levels on the rate of EB1-GFP puncta movement. The majority of data in vertebrate cell cultures systems and in Drosophila S2 cells demonstrate that the EB1 protein itself does not modulate the rate of MT polymerization (Rogers et al., 2002; Tirnauer et al., 2002). We measured the rate of EB1-GFP movement in motor axons using three different GAL4 drivers that have qualitatively different expression levels and different expression profiles (low expression, OK6-GAL4/+; medium expression, D42-GAL4/+; high expression, elaV$\left.G A L 4^{C 155} / Y\right)$. There is no significant difference in the rate of EB1-GFP puncta movement in motor neurons when we compare these three different GAL4 drivers (supplemental Fig. 3, available at www.jneurosci.org as supplemental material). Thus, the EB1GFP puncta movement is independent of EB1-GFP expression levels in three different GAL4 driver lines.

Next, we examined the behavior of dynamic microtubule plus ends marked by GFP within the presynaptic nerve terminal at the NMJ. As a control, we first performed experiments to determine whether EB1-GFP expression alters synapse development at the NMJ. We quantified synaptic bouton numbers and bouton diameter at the third-instar larval neuromuscular junction and find that both parameters are wild type at synapses expressing EB1GFP (bouton number: wt, $15.9 \pm 1.02, n=19$ synapses; UASEB1-GFP, $16.7 \pm 1.05, n=19$ synapses; bouton size: $\mathrm{wt}, 2.22 \pm$ $0.084 \mu \mathrm{m}^{2}, n=191$ boutons; UAS-EB1-GFP, $2.22 \pm 0.80 \mu \mathrm{m}^{2}$, $n=188$ boutons). In addition, synaptic antigens such as nc82 (active zone marker) and anti-Dap160 (periactive zone marker) appear qualitatively normal (supplemental Fig. 2, available at www.jneurosci.org as supplemental material). Because expression of EB1-GFP in vivo does not alter synapse development, we proceeded to use this marker to quantify the behavior of synaptic microtubules in vivo.

In the next series of experiments, we imaged EB1-GFP movement within the synapse and then followed each imaging session by fixation and staining with anti-Futsch. In this way, the move- 
ment of EB1-GFP puncta could be directly compared with the Futsch-positive, bundled MT core (Figs. 5, 6; supplemental Movies 3, 4, respectively, available at www. jneurosci.org as supplemental material). EB1-GFP puncta can be observed to move throughout the synapse. We find that EB1GFP puncta often follow the track of the MT loops identified by anti-Futsch (Fig. $5 B$, arrowheads at right). However, this is not always the case. In some instances, we clearly observe EB1-GFP puncta that traverse across the open center of a MT loop structure, not obeying the route established by anti-Futsch staining (data not shown). Surprisingly, we also observed that individual EB1-GFP puncta are able to take unique paths within a given Futsch loop. In some examples, we observe EB1GFP puncta to travel in opposite directions along a single MT loop simultaneously, crossing paths as they move (Fig. $6 B)$. In other examples, we have seen EB1GFP puncta traverse opposite sides of a single MT loop, traveling in the same direction (Fig. $5 B$, arrowheads, right). Thus, although the majority of EB1-GFP puncta move in the same polarized direction as observed in the axon (plus end away from the soma), the EB1-GFP puncta within the synapse are not restricted to this polarized type of motion. Finally, we quantify the rate of EB1-GFP movement at the synapse (Fig. 5C). The rate of EB1-GFP puncta movement is similar to that observed in the axon (elaV-GAL4 $4^{C 155} / Y$ axon, $4.12 \pm$ $0.038 \mu \mathrm{m} / \mathrm{min}, \quad n=1121$; elaVGAL4 ${ }^{C 155} / Y$ synapse, $4.66 \pm 0.10 \mu \mathrm{m} / \mathrm{min}$, $n=337$; Kolmogorov-Smirnov, $p<0.01$; Student's $t$ test, $p=5.44 \times 10^{-6}$ ).

Next, we asked whether dynamic synaptic pioneer MTs are restricted, in any way, by the presence of the core of bundled MTs labeled by the microtubule-associated protein (MAP) Futsch. Several observations indicate that the EB1-GFP-labeled MTs are independent of MAP stabilized MTs within the presynaptic terminal. First, we find that EB1-GFP puncta can move in regions of the synapse that lack Futsch-positive MTs. We have observed many events in which individual EB1-GFP puncta track the bouton periphery, moving in regions that lack anti-Futsch staining (Fig. 5B, left arrows, and supplemental Movie 3, available at www. jneurosci.org as supplemental material; Fig. 6C). This is nearly always the case within the most distal synaptic bouton in each synapse, called the terminal synaptic bouton (Fig. $5 A, B$; supplemental Movie 3, available at www.jneurosci.org as supplemental material). We also quantified the abundance of EB1-GFP puncta along the NMJ (distal to proximal). Although Futsch labeling tapers dramatically along the length of the NMJ, becoming a fine filament within the terminal bouton (Fig. 5A) (Roos et al., 2000), we do not observe any quantitative change in the abundance of EB1-GFP puncta sampling from proximal and distal regions of the synapse (data not shown). Finally, we examined the behavior of dynamic microtubules in a futsch mutant background that severely disrupts the bundled core filament of MTs normally identified by anti-Futsch staining (Roos et al., 2000). There was no significant change in the rate of EB1-GFP puncta movement in the axons of futsch mutants (Fig. $5 E$ ). We found a small but significant increase in the rate of movement of EB1-GFP at the NMJ of futsch animals (Fig. 5D). Together, these data support the conclusion that EB1-GFP labels an independent population of dynamic pioneer MTs in the axon and presynaptic nerve terminal. The term "dynamic pioneer microtubule" is used to reflect the fact that EB1-GFP puncta identify dynamic MTs (MimoriKiyosue et al., 2000; Rogers et al., 2002; Tirnauer et al., 2002) that are observed to probe regions of the presynaptic nerve terminal that are devoid of stable, bundled microtubules.

\section{Diaphanous modulates the behavior of dynamic pioneer microtubules within the presynaptic nerve terminal} Having used EB1-GFP as a tool for monitoring a dynamic MT population at the synapse in wild-type and futsch mutant animals, we set out to examine this population in dia mutants. We began the analysis of the effect of Dia on dynamic MTs by imag- 

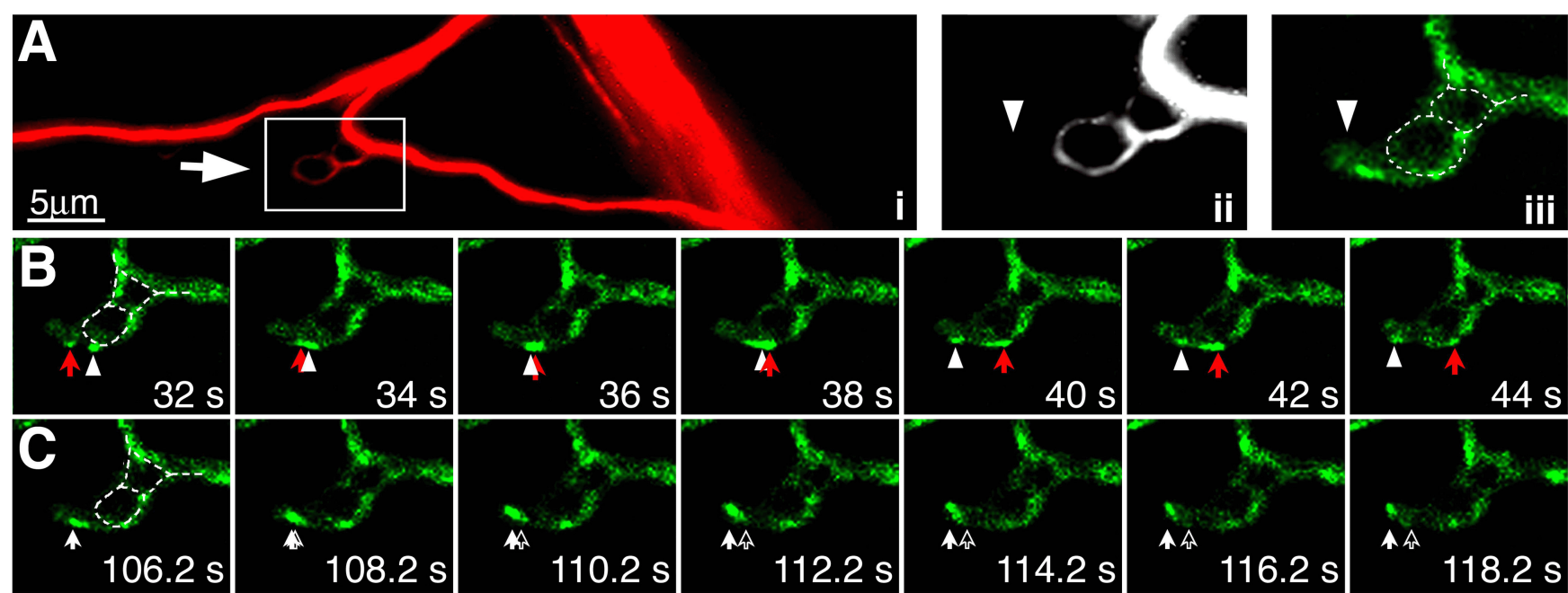

Figure 6. Pioneer microtubule movement relative the Futsch-positive microtubule bundles. $\mathbf{A i}$, Image of the synapse at muscle 4 that was fixed and stained with anti-Futsch to reveal the Futsch-positive MT cytoskeleton. The arrow and box indicate the region of synapse shown at higher magnification to the right. Aii, The portion of the synapse highlighted in Ai shown at higher magnification. Aiii, Before fixation, this synapse was imaged for EB1-GFP puncta movement. EB1-GFP fluorescence is shown within the same region of the synapse highlighted in Ai and Aii, but before fixation. The dotted line demarcates the approximate position of the Futsch-positive MTs based on comparison with Ai and $A$ iii. The arrowhead indicates a prominent EB1-GFP puncta within a region of the nerve terminal that lacks Futsch. $\boldsymbol{B}$, A series of time-lapse images is shown from the highlighted region of the synapse in $\boldsymbol{A}$. The time-lapse series shows the movement of two individual puncta traveling in opposite directions within this bouton. The white arrow indicates a puncta traveling to the left and the red arrow indicates puncta traveling to the right. The dotted line in first image of this series indicates the approximate distribution of anti-Futsch immunoreactivity within the synapse as shown in Aiii. $C$, A different time-lapse series from the same region of the synapse as in $\boldsymbol{A}$ and $\boldsymbol{B}$. In this series, an EB1-GFP puncta moves into a region of the synapse that is devoid of anti-Futsch immunoreactivity. The solid arrow $(t=106.2)$ indicates the starting position of the EB1-GFP puncta in this series. In subsequent images of this series, the hollow arrow demarcates the start position (for reference) and the solid white arrow follows the movement of the EB1-GFP puncta. The dotted line in the first image of this series indicates the distribution Futsch immunoreactivity within the synapse.

ing axons in wild-type and $\mathrm{dia}^{2} / \mathrm{dia}^{2}$ larvae. Time-lapse series were projected to reveal the extent of MT plus end displacement over an identical period of imaging for both genetic backgrounds. We find that the path lengths of EB1-GFP are longer in the $\mathrm{dia}^{2} /$ $\mathrm{dia}^{2}$ background than in wild type (Fig. $7 A, B$, arrowheads), suggesting that MT plus ends are able to move farther in a dia lossof-function background than in a wild-type background over the same time period. To quantify this result, the rate of EB1-GFP puncta movement was measured in both $\mathrm{dia}^{2} / \mathrm{dia}^{2}$ and wild-type larvae. There is a statistically significant shift in the distribution of puncta movement in the dia mutant toward faster rates compared with wild type (Fig. 7C).

We repeated this analysis for EB1-GFP puncta within the presynaptic nerve terminal. As within axons, the path lengths observed in projection images from the $\mathrm{dia}^{2} / \mathrm{dia}^{2}$ synapses are longer than those observed in the wild-type synapses (Fig. 7D, E, arrowheads). Quantification of the rate of EB1-GFP puncta movement again shows a statistically significant shift toward faster rates in the $\mathrm{dia}^{2} / \mathrm{dia}^{2}$ animals compared with wild type (Fig. $7 F$ ). Together, the increased rate of movement seen in axon and the synapse implies that loss of Diaphanous leads to an increase in the rate of MT polymerization. The length of EB1-comet tails has been used in other studies as a means of measuring differences in rates of polymerization. Because the comet tail is thought to arise from the release of EB1 molecules as the GTP-cap is hydrolyzed, a longer comet tail represents an increase in polymerization. We measured and compared the length of the EB1-GFP comet tails in $\mathrm{dia}^{2} / \mathrm{dia}^{2}$ and wild-type axons. Consistent with the increased rate of movement, the comet tails in the $\mathrm{dia}^{2} / \mathrm{dia}^{2}$ larvae are twofold longer than those in wild type (wt, $0.31 \pm 0.006 \mu \mathrm{m} ; \mathrm{dia}^{2 / 2}$, $\left.0.565 \pm 0.006 \mu \mathrm{m} ; p=9.7 \mathrm{E}^{-129}\right)$.

Finally, we asked whether the regulation of dynamic MTs by Diaphanous could be generalized to other Drosophila cell types. To do so, we used gene-specific RNAi to dia to deplete Dia pro- tein from S2 cells. Western blots revealed that Diaphanous protein level was absent after $5 \mathrm{~d}$ of dsRNA treatment (data not shown). Previous studies in which Dia has been knocked down with RNAi have shown that S2 cell size increases and the cells become multinucleate because of a failure to undergo cytokinesis. In our study, we noted that cells treated with dsRNA against diaphanous showed these same phenotypes. After Dia RNAi, EB1-GFP puncta have longer path lengths relative to the control cells, consistent with the results from the axon and synapse (Fig. $7 G, H)$. A histogram of the rate of EB1-GFP puncta movement in both the RNAi-treated and control cells reveals a statistically significant shift toward faster puncta movement in diaphanous RNAi cells (Fig. 7I).

One possibility is that the Dia-dependent change in synaptic actin leads to a change in the rate of MT plus end movement. To test this possibility, we pharmacologically depolymerized synaptic actin and assayed MT plus end movement within the presynaptic nerve terminal. We find that synaptic actin foci were completely eliminated by incubation in $7.5 \mu \mathrm{M}$ latrunculin A (Fig. $8 A, B)$. We then imaged MT plus end movement after this treatment. We find that loss of actin foci has no direct effect on MT plus end movement compared with controls (Fig. 8C-E). Therefore, together with our in vivo and in vitro imaging data, our results are consistent with the conclusion that Diaphanous participates in the regulation of MT plus end polymerization within the axon and the presynaptic nerve terminal. These data are consistent with results from other systems indicating that Dia has a stabilizing activity at MT plus ends.

It is possible that the change in MT plus end behavior has a direct effect on synaptic growth. MT dynamic instability is essential for growth cone and cell motility (Rodriguez et al., 2003; Lee et al., 2004; Suter et al., 2004) and has been proposed to be involved in the detection or relay of signaling events at the cell plasma membrane (Kalil and Dent, 2005). Another possibility is 
that the change in MT plus end behavior results in a defect in the assembly or organization of the underlying stable MT population identified by anti-Futsch staining. Therefore, we quantified Futsch staining and compared wild type and dia mutants. We find that Futch-positive MTs remain well organized in the dia mutant background (Fig. 9A-C). However, closer examination reveals a modest, although statistically significant, decrease in the abundance of Futsch-positive MTs at the distal-most regions of the NMJ. In wildtype animals, the caliber of the Futschpositive MT filament decreases along the length of the NMJ until it becomes a very fine filament in the most distal bouton. In dia mutant NMJ, the Futsch-positive MTs taper more rapidly within the NMJ (Fig. $9 B, C)$. As a consequence, the distal regions of the dia mutant NMJ appear to lack a clear core of bundled MTs. Quantitatively identical results were obtained imaging Futsch-positive MTs in the dlar mutant and the dlar; dia double mutant ( $p<$ 0.01) (data not shown). This effect in the dia mutant is not caused by decreased numbers of pioneer MTs in distal regions of the NMJ (Fig. 9D,E). When we quantify the number of EB1-GFP plus ends in the distal $50 \mu \mathrm{m}$ of the NMJ, there is no statistically significant difference comparing wild type and $\mathrm{dia}^{2} / \mathrm{dia}^{2}$ (plus ends in distal $50 \mu \mathrm{m}$ of NMJ; elaV ${ }^{\mathrm{C155}}$; UAS-EB1$G F P /+, 20 \pm 2.5$, compared with ela $V^{C 155}$; $\mathrm{dia}^{2} ; U A S-E B 1-G F P /+, 22.8 \pm 2.0 ; p>$ $0.3)$. Therefore, we speculate that altered behavior of these pioneer microtubules could be related to the impaired incorporation/stabilization of new MTs into the bundled, MAP-positive core of MTs. Live imaging studies have shown that NMJ growth can be achieved, in part, through the addition of new synaptic boutons at the distal end of the NMJ (Zito et al., 1999). Because the integrity of the Futsch-positive MTs are necessary for synaptic growth, it is possible that this defect observed in the dia and dlar mutants contributes to defective synaptic growth.

\section{Discussion}

Here, we advance our understanding of synaptic growth and development in several fundamental ways. In a forward genetic screen, we identified Diaphanous as an important regulator of synaptic growth. Immunohistochemical data as well as several independent genetic experiments demonstrate that Dia functions presynaptically to influence synaptic growth. We then provide evidence that Dia is necessary for the normal regulation of both the presynaptic actin and MT cytoskeletons, including a population of newly characterized dynamic pioneer MTs. Finally, we provide genetic evidence that Dia functions downstream of the receptor tyrosine phosphatase Dlar. Based on our data, we propose that Dia functions downstream of Dlar and Trio to influence either the formation or maintenance of the synaptic actin and MT cytoskeletons. In this way, Dia represents an essential effector that could couple intercellular growth-related signaling at the synaptic membrane to the regulation of synaptic actin and a dynamic population of synaptic microtubules. It has been proposed that formin proteins such as Dia may be able to coordinate the regulation of the actin and MT cytoskeletons. Although our data do not directly address this topic, it is interesting to speculate that synaptic growth might require both actin-mediated membrane extension and a coordinate MT-dependent stabilization of newly formed synaptic boutons.

\section{Evidence that Dia functions downstream of Dlar and Trio to control synaptic growth}

A genetic epistasis analysis provides evidence that Dia functions in the same genetic pathway as Dlar and Trio to control synaptic growth. There are strong transheterozygous interactions of the dia null mutation with mutations in both dlar and trio. In addition, synaptic growth defects in the dia; dlar double mutant are no more severe than that observed in either single mutation alone. The placement of Dia downstream of Dlar is supported by the demonstration that neuronal expression of an activated diaphanous transgene rescues NMJ morphology and animal health in the dlar mutant background. Furthermore, the placement of Dia downstream of Trio is consistent with the proposed function 

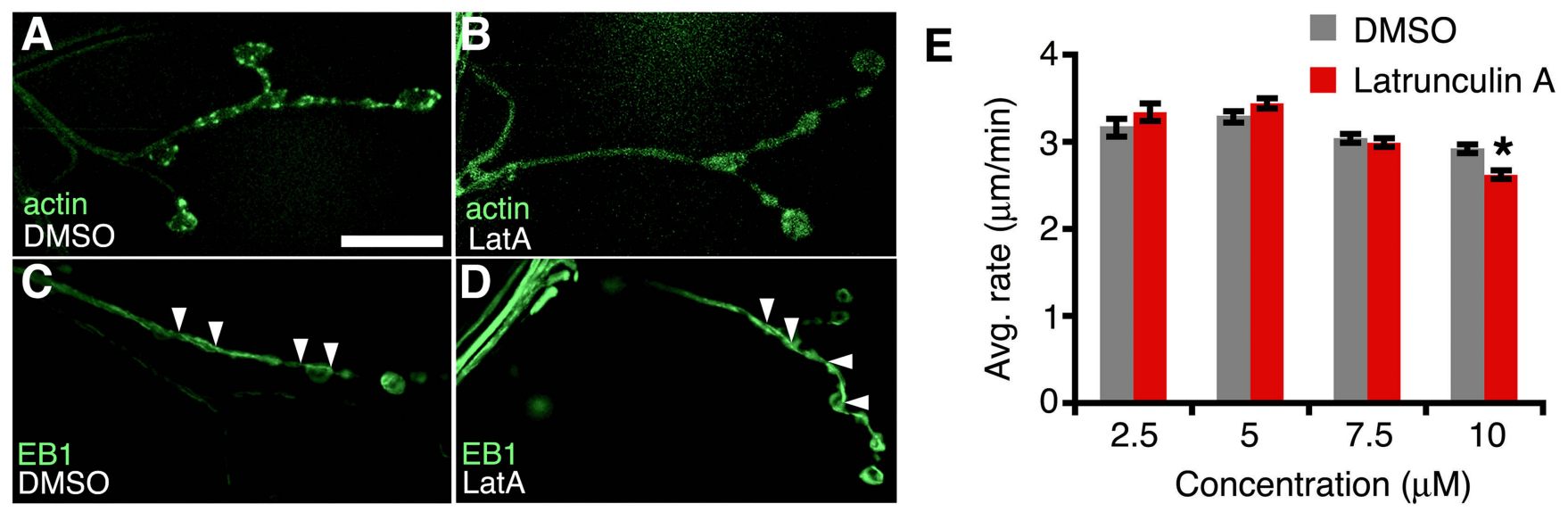

Figure 8. Behavior of synaptic microtubule plus ends is not altered by disruption of synaptic actin. $A, B$, Representative images of third-instar $m 4$ NMJs in animals expressing UAS-GFP-actin driven by elavGAL4 $4^{(155}$ incubated in vehicle (DMSO) alone $(\boldsymbol{A})$ or in $7.5 \mu \mathrm{m}$ latrunculin $\mathrm{A}(\boldsymbol{B})$. C, $\boldsymbol{D}$, Representative projections of time-lapse images at $\mathrm{m} 4$ in animals expressing UAS-EBT-GFP driven by elavGAL4 ${ }^{1155}$ incubated in vehicle alone $(\boldsymbol{C})$ or in $7.5 \mu \mathrm{m}$ latrunculin $\mathrm{A}(\boldsymbol{D})$. The arrowheads highlight the paths of individual EB1-GFP puncta over the same time frame. Scale bar, $5 \mu \mathrm{m}$. $\boldsymbol{E}$, Average EB1-GFP puncta velocities in DMSO (gray bars)- and latrunculin A (red bars)-treated animals for the concentrations tested (2.5 $\mu \mathrm{m}: \mathrm{DMSO}, 3.18 \pm 0.08 \mu \mathrm{m} / \mathrm{min}, n=194 ; \mathrm{LatA}, 3.34 \pm 0.1 \mu \mathrm{m} / \mathrm{min}$, $n=136 ; p=0.23 ; 5 \mu \mathrm{M}:$ DMSO, $3.3 \pm 0.05 \mu \mathrm{m} / \mathrm{min}, n=351 ;$ LatA, $3.44 \pm 0.06 \mu \mathrm{m} / \mathrm{min}, n=340 ; p=0.07 ; 7.5 \mu \mathrm{m}:$ DMSO, $3.04 \pm 0.05 \mu \mathrm{m} / \mathrm{min}, n=368 ;$ LatA, $2.99 \pm 0.05 \mu \mathrm{m} / \mathrm{min}$, $n=291 ; p=0.57 ; 10 \mu \mathrm{m}:$ DMSO, $\left.2.92 \pm 0.05 \mu \mathrm{m} / \mathrm{min}, n=254 ; \mathrm{LatA}, 2.62 \pm 0.05 \mu \mathrm{m} / \mathrm{min}, n=217 ; p=3.6 \times 10^{-5}\right)$. There is no significant difference in the average puncta velocity at most of the concentrations tested. At $10 \mu \mathrm{m}$ latrunculin A, EB1-GFP movement is slightly but significantly ${ }^{*}$ ) slower than vehicle alone. Statistical significance was determined by Student's $t$ test. Error bars represent SEM.
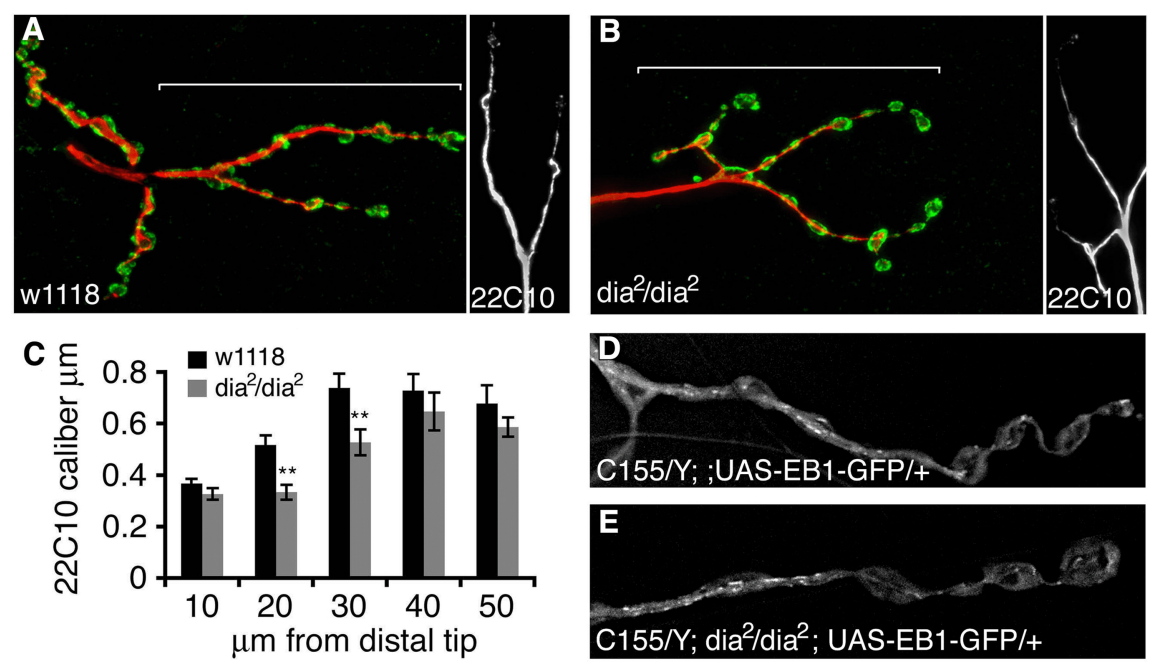

Figure 9. The density of stable microtubules is disrupted at dia mutant synapses. $A, B$, Immunofluorescent image of thirdinstar NMJ at muscle 4 from wild-type $(\boldsymbol{A})$ and $\mathrm{dia}^{2} / \mathrm{dia}^{2}(\boldsymbol{B})$ larvae. Stable microtubules labeled by the $22 \mathrm{C} 10$ antibody are shown in red; presynaptic membrane is labeled with the HRP antibody shown in green. Scale bar, $10 \mu \mathrm{m}$. Shown next to each panel is the magnified and isolated $22 \mathrm{C} 10$ staining from the distal $50 \mu \mathrm{m}$ of each NMJ (rotated $90^{\circ}$ counterclockwise). The region shown in inset is highlighted by brackets in the image at left. C, Quantification of $22 \mathrm{C} 10$ average staining intensity in wild-type and $\mathrm{dia}^{2} / \mathrm{dia}^{2}$ $\mathrm{m} 4$ synapses. Error bars represent SEM. $\boldsymbol{D}, \boldsymbol{E}$, EB1-GFP puncta are shown in a projected image of wild type $(\boldsymbol{D})$ and $\mathrm{dia}^{2} / \mathrm{dia}^{2}(\boldsymbol{E})$ over a 30 s interval. Asterisks indicate statistically significant differences compared with wild type.

of Diaphanous-related formin proteins as Rho-type GTPase effectors that link signaling at the plasma membrane to the regulated modulation of the underlying cytoskeleton (Palazzo et al., 2004; Wen et al., 2004; Pellegrin and Mellor, 2005). Dlar has been implicated in the mechanisms of synaptic growth in organisms ranging from Caenorhabditis elegans to Drosophila and mammals (Kaufmann et al., 2002; Wyszynski et al., 2002; Dunah et al., 2005). For example, disruption of mammalian Lar signaling has been shown to significantly alter synapse and spine morphogenesis in the vertebrate CNS (Wyszynski et al., 2002; Dunah et al., 2005; Hoogenraad et al., 2007). However, the means by which Lar might influence the synaptic cytoskeleton were not addressed in these studies. By providing a molecular link between Dlar and the underlying cytoskeleton, Dia may provide an important advance in dissecting the complex function of Lar signaling, and formin signaling, in the nervous system.

Current evidence indicates that Dlardependent control of neural development is complex. In systems ranging from $C$. elegans to mammals, Dlar has been shown to interact both biochemically and genetically with the cytoplasmic protein Liprin- $\alpha$ (Zhen and Jin, 1999; Kaufmann et al., 2002; Wyszynski et al., 2002; Dunah et al., 2005; Hoogenraad et al., 2007). Liprin- $\alpha$ may be responsible for the regulated trafficking of Dlar to specific sites within the cell (Shin et al., 2003) and thereby influence synapse development. However, genetic analyses demonstrate that Liprin- $\alpha$-dependent signaling mediates effects that are more extensive than can be accounted for by loss of dlar alone. For example, liprin- $\alpha$ mutations strongly perturb active-zone architecture and neurotransmission, whereas dlar mutations have milder effects on active-zone organization and function (Kaufmann et al., 2002). It seems, based on current evidence from diverse systems, that Liprin- $\alpha$ function extends to influence the synaptic particle web in a manner that is independent of DLar function (Zhen and Jin, 1999; Kaufmann et al., 2002; Wyszynski et al., 2002; Dunah et al., 2005)

Recently, two studies independently identified ligands for the Dlar receptor in Drosophila (Fox and Zinn, 2005; Johnson et al., 2006). At the Drosophila NMJ, Dlar binds to the HSPGs (heparin sulfate proteoglycans) Dally-like (Dlp) and Syndecan (Sdc) (Johnson et al., 2006). Current analysis indicates that Dlp inhibits Dlar function, whereas Sdc activates Dlar. Interestingly, mutation of Sdc phenocopies the defect in synaptic growth observed in the Dlar mutant, but $s d c$ mutants do not have a defect in synaptic transmission, active-zone structure, or the distribution of synaptic markers (John- 
son et al., 2006). Thus, although the regulation of Dlar-mediated signaling may be quite complex, it is possible that the Dlardependent synaptic growth is mediated by a specific set of signaling interactions that include the ligand Sdc and downstream signaling mediated by Trio and Diaphanous.

In Drosophila, unlike mammalian systems, Dlar is specifically presynaptic. Consistently, Dia functions presynaptically to specify NMJ growth and regulation of the presynaptic cytoskeleton. Thus, the Dia-Dlar genetic interaction presumably reflects the action of this signaling pathway in the presynaptic nerve terminal. In other systems, such as mammalian central synapses, LAR is present on both sides of the synaptic terminal and defects in both AMPA receptor clustering and synapse morphogenesis are observed (Wyszynski et al., 2002). It is interesting to speculate that Dia may be involved in synapse morphogenesis downstream of LAR, whereas liprin- $\alpha$ related signaling is involved in the control of AMPA receptor abundance and active-zone integrity.

\section{Synaptic pioneer MTs and the control of synapse morphology} The importance of the synaptic MT cytoskeleton during synaptic development and neurological disease has been highlighted by many studies (Zhang and Broadie, 2005). In particular, essential MT regulatory proteins are linked to diverse neurological disease including spastic paraplegia and fragile X syndrome (Sherwood et al., 2004; Roll-Mecak and Vale, 2005; Zhang and Broadie, 2005). Although defects in the MAP-stabilized MTs have been interpreted to reflect changes in MT dynamics (Roos et al., 2000; Ruiz-Canada et al., 2004), there is no evidence that the Futsch-stabilized MTs represent a dynamic population of MTs. By labeling MT plus ends with EB1GFP, we are able to characterize a population of dynamic pioneer MTs at the Drosophila NMJ, opening the door to a detailed characterization of MT regulation during synaptic growth and models of neuronal disease.

There are two possible models for how Dia-dependent modulation of pioneer MTs could influence synaptic growth. Dia and other formins have been implicated in MT stabilization through plus end binding (Palazzo et al., 2004). We find that pioneer MTs are evenly distributed throughout the NMJ and probe the cortex of the presynaptic membrane. One possibility is that Dia-dependent stabilization of the pioneer MT plus ends is involved in relaying signaling from the plasma membrane to the MT cytoskeleton. Consistent with this hypothesis is the observation that MT plus end movement is more rapid in dia mutants, potentially disrupting the signaling relay between plasma membrane signaling complexes and the dynamic microtubule plus end. Alternatively, Dia-dependent modulation of pioneer MT behavior could influence the consolidation of dynamic MTs into the MAP-stabilized, Futsch-positive core. Impairment of this process could disrupt NMJ growth. This model is similar to the consolidation of dynamic peripheral MTs into a MAP-stabilized central domain of the growth cone, a process necessary for growth cone advance (Rodriguez et al., 2003). In either case, our data open up a new avenue of investigation for synaptic development at the Drosophila NMJ.

\section{References}

Aberle H, Haghighi AP, Fetter RD, McCabe BD, Magalhães TR, Goodman CS (2002) Wishful thinking encodes a BMP type II receptor that regulates synaptic growth in Drosophila. Neuron 33:545-558.

Alberts AS (2001) Identification of a carboxyl-terminal diaphanous-related formin homology protein autoregulatory domain. J Biol Chem 276:2824-2830.

Bamji SX, Rico B, Kimes N, Reichardt LF (2006) BDNF mobilizes synaptic vesicles and enhances synapse formation by disrupting cadherin-betacatenin interactions. J Cell Biol 174:289-299.

Bateman J, Shu H, Van Vactor D (2000) The guanine nucleotide exchange factor trio mediates axonal development in the Drosophila embryo. Neuron 26:93-106.

Castrillon DH, Wasserman SA (1994) Diaphanous is required for cytokinesis in Drosophila and shares domains of similarity with the products of the limb deformity gene. Development 120:3367-3377.

Coyle IP, Koh YH, Lee WC, Slind J, Fergestad T, Littleton JT, Ganetzky B (2004) Nervous wreck, an SH3 adaptor protein that interacts with Wsp, regulates synaptic growth in Drosophila. Neuron 41:521-534.

Dunah AW, Hueske E, Wyszynski M, Hoogenraad CC, Jaworski J, Pak DT, Simonetta A, Liu G, Sheng M (2005) LAR receptor protein tyrosine phosphatases in the development and maintenance of excitatory synapses. Nat Neurosci 8:458-467.

Eaton BA, Davis GW (2005) LIM Kinasel controls synaptic stability downstream of the type II BMP receptor. Neuron 47:695-708.

Eaton BA, Fetter RD, Davis GW (2002) Dynactin is necessary for synapse stabilization. Neuron 34:729-741.

Edwards KA, Demsky M, Montague RA, Weymouth N, Kiehart DP (1997) GFP-moesin illuminates actin cytoskeleton dynamics in living tissue and demonstrates cell shape changes during morphogenesis in Drosophila. Dev Biol 191:103-117.

Emmons S, Phan H, Calley J, Chen W, James B, Manseau L (1995) Cappuccino, a Drosophila maternal effect gene required for polarity of the egg and embryo, is related to the vertebrate limb deformity locus. Genes Dev 9:2482-2494.

Eng CH, Huckaba TM, Gundersen GG (2006) The formin mDia regulates GSK3beta through novel PKCs to promote microtubule stabilization but not MTOC reorientation in migrating fibroblasts. Mol Biol Cell 17:5004-5016.

Fox AN, Zinn K (2005) The heparan sulfate proteoglycan syndecan is an in vivo ligand for the Drosophila LAR receptor tyrosine phosphatase. Curr Biol 15:1701-1711.

Higgs HN (2005) Formin proteins: a domain-based approach. Trends Biochem Sci 30:342-353.

Hoogenraad CC, Feliu-Mojer MI, Spangler SA, Milstein AD, Dunah AW, Hung AY, Sheng M (2007) Liprinalphal degradation by calcium/calmodulindependent protein kinase II regulates LAR receptor tyrosine phosphatase distribution and dendrite development. Dev Cell 12:587-602.

Ishizaki T, Morishima Y, Okamoto M, Furuyashiki T, Kato T, Narumiya S (2001) Coordination of microtubules and the actin cytoskeleton by the Rho effector mDial. Nat Cell Biol 3:8-14.

Johnson KG, Tenney AP, Ghose A, Duckworth AM, Higashi ME, Parfitt K, Marcu O, Heslip TR, Marsh JL, Schwarz TL, Flanagan JG, Van Vactor D (2006) The HSPGs Syndecan and Dallylike bind the receptor phosphatase LAR and exert distinct effects on synaptic development. Neuron 49:517-531.

Kalil K, Dent EW (2005) Touch and go: guidance cues signal to the growth cone cytoskeleton. Curr Opin Neurobiol 15:521-526.

Kaufmann N, DeProto J, Ranjan R, Wan H, Van Vactor D (2002) Drosophila liprin-alpha and the receptor phosphatase Dlar control synapse morphogenesis. Neuron 34:27-38.

Kobielak A, Pasolli HA, Fuchs E (2004) Mammalian formin-1 participates in adherens junctions and polymerization of linear actin cables. Nat Cell Biol 6:21-30.

Kovar DR, Pollard TD (2004) Insertional assembly of actin filament barbed ends in association with formins produces piconewton forces. Proc Natl Acad Sci U S A 101:14725-14730.

Kovar DR, Kuhn JR, Tichy AL, Pollard TD (2003) The fission yeast cytokinesis formin Cdc12p is a barbed end actin filament capping protein gated by profilin. J Cell Biol 161:875-887.

Krueger NX, Van Vactor D, Wan HI, Gelbart WM, Goodman CS, Saito H (1996) The transmembrane tyrosine phosphatase DLAR controls motor axon guidance in Drosophila. Cell 84:611-622.

Lee H, Engel U, Rusch J, Scherrer S, Sheard K, Van Vactor D (2004) The microtubule plus end tracking protein Orbit/MAST/CLASP acts downstream of the tyrosine kinase Abl in mediating axon guidance. Neuron 42:913-926.

Li F, Higgs HN (2003) The mouse Formin mDial is a potent actin nucleation factor regulated by autoinhibition. Curr Biol 13:1335-1340.

Li F, Higgs HN (2005) Dissecting requirements for auto-inhibition of actin nucleation by the formin, mDia1. J Biol Chem 280:6986-6992.

Liebl FL, Chen K, Karr J, Sheng Q, Featherstone DE (2005) Increased synaptic microtubules and altered synapse development in Drosophila sec8 mutants. BMC Biol 3:27.

Luo L, O'Leary DD (2005) Axon retraction and degeneration in development and disease. Annu Rev Neurosci 28:127-156. 
Ma Y, Shakiryanova D, Vardya I, Popov SV (2004) Quantitative analysis of microtubule transport in growing nerve processes. Curr Biol 14:725-730.

Marie B, Sweeney ST, Poskanzer KE, Roos J, Kelly RB, Davis GW (2004) Dap160/intersectin scaffolds the periactive zone to achieve high-fidelity endocytosis and normal synaptic growth. Neuron 43:207-219.

Marqués G (2005) Morphogens and synaptogenesis in Drosophila. J Neurobiol 64:417-434.

Mimori-Kiyosue Y, Shiina N, Tsukita S (2000) The dynamic behavior of the APC-binding protein EB1 on the distal ends of microtubules. Curr Biol 10:865-868.

Morrison EE, Wardleworth BN, Askham JM, Markham AF, Meredith DM (1998) EB1, a protein which interacts with the APC tumour suppressor, is associated with the microtubule cytoskeleton throughout the cell cycle. Oncogene 17:3471-3477.

Morrison EE, Moncur PM, Askham JM (2002) EB1 identifies sites of microtubule polymerisation during neurite development. Brain Res Mol Brain Res 98:145-152.

Newsome TP, Schmidt S, Dietzl G, Keleman K, Asling B, Debant A, Dickson BJ (2000) Trio combines with dock to regulate Pak activity during photoreceptor axon pathfinding in Drosophila. Cell 101:283-294.

Nunes P, Haines N, Kuppuswamy V, Fleet DJ, Stewart BA (2006) Synaptic vesicle mobility and presynaptic F-actin are disrupted in a $\mathrm{N}$-ethylmaleimidesensitive factor allele of Drosophila. Mol Biol Cell 17:4709-4719.

Palazzo AF, Cook TA, Alberts AS, Gundersen GG (2001) mDia mediates Rho-regulated formation and orientation of stable microtubules. Nat Cell Biol 3:723-729.

Palazzo AF, Eng CH, Schlaepfer DD, Marcantonio EE, Gundersen GG (2004) Localized stabilization of microtubules by integrin- and FAKfacilitated Rho signaling. Science 303:836-839.

Pellegrin S, Mellor H (2005) The Rho family GTPase Rif induces filopodia through mDia2. Curr Biol 15:129-133.

Pennetta G, Hiesinger PR, Fabian-Fine R, Meinertzhagen IA, Bellen H) (2002) Drosophila VAP-33A directs bouton formation at neuromuscular junctions in a dosage-dependent manner. Neuron 35:291-306.

Piehl M, Tulu US, Wadsworth P, Cassimeris L (2004) Centrosome maturation: measurement of microtubule nucleation throughout the cell cycle by using GFP-tagged EB1. Proc Natl Acad Sci U S A 101:1584-1588.

Pielage J, Fetter RD, Davis GW (2005) Presynaptic spectrin is essential for synapse stabilization. Curr Biol 15:918-928.

Pielage J, Fetter RD, Davis GW (2006) A postsynaptic spectrin scaffold defines active zone size, spacing, and efficacy at the Drosophila neuromuscular junction. J Cell Biol 175:491-503.

Pruyne D, Evangelista M, Yang C, Bi E, Zigmond S, Bretscher A, Boone C (2002) Role of formins in actin assembly: nucleation and barbed-end association. Science 297:612-615.

Reichardt LF (2006) Neurotrophin-regulated signalling pathways. Philos Trans R Soc Lond B Biol Sci 361:1545-1564.

Ridley AJ (2006) Rho GTPases and actin dynamics in membrane protrusions and vesicle trafficking. Trends Cell Biol 16:522-529.

Rodriguez OC, Schaefer AW, Mandato CA, Forscher P, Bement WM Waterman-Storer CM (2003) Conserved microtubule-actin interactions in cell movement and morphogenesis. Nat Cell Biol 5:599-609.

Rogers SL, Rogers GC, Sharp DJ, Vale RD (2002) Drosophila EB1 is important for proper assembly, dynamics, and positioning of the mitotic spindle. J Cell Biol 158:873-884.

Roll-Mecak A, Vale RD (2005) The Drosophila homologue of the hereditary spastic paraplegia protein, spastin, severs and disassembles microtubules. Curr Biol 15:650-655.

Rolls MM, Satoh D, Clyne PJ, Henner AL, Uemura T, Doe CQ (2007) Polarity and intracellular compartmentalization of Drosophila neurons. Neural Develop 2:7.

Romero S, Le Clainche C, Didry D, Egile C, Pantaloni D, Carlier MF (2004) Formin is a processive motor that requires profilin to accelerate actin assembly and associated ATP hydrolysis. Cell 119:419-429.

Roos J, Hummel T, Ng N, Klämbt C, Davis GW (2000) Drosophila Futsch regulates synaptic microtubule organization and is necessary for synaptic growth. Neuron 26:371-382.

Ruiz-Canada C, Ashley J, Moeckel-Cole S, Drier E, Yin J, Budnik V (2004) New synaptic bouton formation is disrupted by misregulation of microtubule stability in aPKC mutants. Neuron 42:567-580.

Sagot I, Rodal AA, Moseley J, Goode BL, Pellman D (2002) An actin nucleation mechanism mediated by Bnil and profilin. Nat Cell Biol 4:626-631.
Schuyler SC, Pellman D (2001) Microtubule "plus-end-tracking proteins": the end is just the beginning. Cell 105:421-424.

Sherwood NT, Sun Q, Xue M, Zhang B, Zinn K (2004) Drosophila spastin regulates synaptic microtubule networks and is required for normal motor function. PLoS Biol 2:e429.

Shin H, Wyszynski M, Huh KH, Valtschanoff JG, Lee JR, Ko J, Streuli M, Weinberg RJ, Sheng M, Kim E (2003) Association of the kinesin motor KIF1A with the multimodular protein liprin-alpha. J Biol Chem 278:11393-11401.

Somogyi K, Rørth P (2004) Evidence for tension-based regulation of Drosophila MAL and SRF during invasive cell migration. Dev Cell 7:85-93.

Stepanova T, Slemmer J, Hoogenraad CC, Lansbergen G, Dortland B, De Zeeuw CI, Grosveld F, van Cappellen G, Akhmanova A, Galjart N (2003) Visualization of microtubule growth in cultured neurons via the use of EB3-GFP (end-binding protein 3-green fluorescent protein). J Neurosci 23:2655-2664.

Stewart BA, Mohtashami M, Rivlin P, Deitcher DL, Trimble WS, Boulianne GL (2002) Dominant-negative NSF2 disrupts the structure and function of Drosophila neuromuscular synapses. J Neurobiol 51:261-271.

Stewart BA, Pearce J, Bajec M, Khorana R (2005) Disruption of synaptic development and ultrastructure by Drosophila NSF2 alleles. J Comp Neurol 488:101-111.

Suter DM, Schaefer AW, Forscher P (2004) Microtubule dynamics are necessary for SRC family kinase-dependent growth cone steering. Curr Biol 14:1194-1199.

Tanaka E, Ho T, Kirschner MW (1995) The role of microtubule dynamics in growth cone motility and axonal growth. J Cell Biol 128:139-155.

Tirnauer JS, Grego S, Salmon ED, Mitchison TJ (2002) EB1-microtubule interactions in Xenopus egg extracts: role of EB1 in microtubule stabilization and mechanisms of targeting to microtubules. Mol Biol Cell 13:3614-3626.

Tominaga T, Sahai E, Chardin P, McCormick F, Courtneidge SA, Alberts AS (2000) Diaphanous-related formins bridge Rho GTPase and Src tyrosine kinase signaling. Mol Cell 5:13-25.

Viquez NM, Li CR, Wairkar YP, DiAntonio A (2006) The B' protein phosphatase $2 \mathrm{~A}$ regulatory subunit well-rounded regulates synaptic growth and cytoskeletal stability at the Drosophila neuromuscular junction. J Neurosci 26:9293-9303.

Wallar BJ, Alberts AS (2003) The formins: active scaffolds that remodel the cytoskeleton. Trends Cell Biol 13:435-446.

Wallar BJ, Stropich BN, Schoenherr JA, Holman HA, Kitchen SM, Alberts AS (2006) The basic region of the diaphanous-autoregulatory domain (DAD) is required for autoregulatory interactions with the diaphanousrelated formin inhibitory domain. J Biol Chem 281:4300-4307.

Watanabe N, Kato T, Fujita A, Ishizaki T, Narumiya S (1999) Cooperation between mDial and ROCK in Rho-induced actin reorganization. Nat Cell Biol 1:136-143.

Wen Y, Eng CH, Schmoranzer J, Cabrera-Poch N, Morris EJ, Chen M, Wallar BJ, Alberts AS, Gundersen GG (2004) EB1 and APC bind to mDia to stabilize microtubules downstream of Rho and promote cell migration. Nat Cell Biol 6:820-830.

Williams MJ, Habayeb MS, Hultmark D (2007) Reciprocal regulation of Racl and Rho1 in Drosophila circulating immune surveillance cells. J Cell Sci 120:502-511.

Wyszynski M, Kim E, Dunah AW, Passafaro M, Valtschanoff JG, Serra-Pagès C, Streuli M, Weinberg RJ, Sheng M (2002) Interaction between GRIP and liprin-alpha/SYD2 is required for AMPA receptor targeting. Neuron 34:39-52.

Yan Y, Broadie K (2007) In vivo assay of presynaptic microtubule cytoskeleton dynamics in Drosophila. J Neurosci Methods 162:198-205.

Zhang YQ, Broadie K (2005) Fathoming fragile X in fruit flies. Trends Genet 21:37-45.

Zhen M, Jin Y (1999) The liprin protein SYD-2 regulates the differentiation of presynaptic termini in C. elegans. Nature 401:371-375.

Zigmond SH (2004) Formin-induced nucleation of actin filaments. Curr Opin Cell Biol 16:99-105.

Zigmond SH, Evangelista M, Boone C, Yang C, Dar AC, Sicheri F, Forkey J, Pring M (2003) Formin leaky cap allows elongation in the presence of tight capping proteins. Curr Biol 13:1820-1823.

Zito K, Parnas D, Fetter RD, Isacoff EY, Goodman CS (1999) Watching a synapse grow: noninvasive confocal imaging of synaptic growth in Drosophila. Neuron 22:719-729.

Zweifel LS, Kuruvilla R, Ginty DD (2005) Functions and mechanisms of retrograde neurotrophin signalling. Nat Rev Neurosci 6:615-625. 